\title{
Evolution and growth of the ECHO (Enriching Communication skills for Health professionals in Oncofertility) program: a 5-year study in the training of oncofertility professionals
}

\author{
Jillian Pecoriello ${ }^{1}$ - James L. Klosky ${ }^{4}$ Bianca Augusto ${ }^{2} \cdot$ Waleska Santiago-Datil $^{2}$. Amani Sampson ${ }^{1}$. \\ Richard Reich $^{3}$. Susan Vadaparampil ${ }^{2}$. Gwendolyn Quinn ${ }^{1}$
}

Received: 24 July 2021 / Accepted: 13 November 2021

(c) The Author(s), under exclusive licence to Springer Science+Business Media, LLC, part of Springer Nature 2021

\begin{abstract}
Purpose AYAs with cancer have unique psychosocial needs, with reproductive health being a primary concern. The ECHO training program provides reproductive health communication training to individuals providing care for AYAs with cancer. The purpose of this project is to describe the growth of ECHO and evaluate changes in learner engagement over a 5-year period.

Methods ECHO is an 8-week online training program offered annually, with the program including learning modules, discussion topics and reflections, and synchronous discussions. Reflection quality scores and number of words were compared between the 5 cohorts using ANOVA with a $p<.05$ level of significance. Descriptive statistics summarized module topics, reflections, and synchronous discussions.

Results The average number of reflections per unique learner increased each year (1.4 in cohort 1 vs 4.1 in cohort 5), as did average length and quality of reflections (72.1 words in cohort 1 vs 203.4 words in cohort 5, $p<.0001$; score of 1.21 in cohort 1 vs 4.46 in cohort $5, p<.0001)$. The percentage of learners in attendance at synchronous discussions increased between cohorts 4 and 5 (4.8\% of learners in cohort 4 vs $18.8 \%$ of learners cohort 5$)$.

Conclusions The ECHO program has seen significant growth and improvement in learner engagement over a 5-year period. This is particularly important given that student learning outcomes in online courses can be predicted by the level of engagement with online content.

Implications for Cancer Survivors As fertility and reproductive health remain a top life goal and discussion priority for AYAs surviving cancer, increasing clinical competencies of AHPs in oncofertility is essential.
\end{abstract}

Keywords Reproductive health $\cdot$ Oncofertility $\cdot$ Education Intervention $\cdot$ AYA

\section{Introduction}

Jillian Pecoriello

Jillian.Pecoriello@nyulangone.org

1 Department of Obstetrics and Gynecology, New York University, New York, NY, USA

2 Health Outcomes and Behavior Program, Moffitt Cancer Center, Tampa, FL, USA

3 Biostatistics and Bioinformatics Shared Resource, Moffitt Cancer Center, Tampa, FL, USA

4 Department of Pediatrics, Emory University School of Medicine \& Children's Healthcare of Atlanta, Atlanta, GA, USA
Supportive medical care, in the form of effective communication and expedient dissemination of information, is one of the most important factors in the overall quality of care experience for adolescents and young adults (AYAs) with cancer $[1,2]$. AYA patients are a psychosocially at-risk group and consistently report reproductive health as being a primary concern in survivorship. One study found that having children was ranked within the top 3 overall life goals of newly diagnosed AYA males, while in another, all AYA females with cancer reported a strong desire for biological children in the future [3, 4]. Gorman et al. identified common themes in discussions about fertility among AYA female cancer survivors, including finding that all participants in the study 
desired more information about fertility from a healthcare provider. Additionally, participants reported feeling disappointed that many of their providers wanted to delay fertility discussions until they were ready to have children. The majority of participants identified that the reason for wanting to have more information about their fertility was to plan and prepare for the future [5]. Furthermore, in a survey of AYA cancer survivors, $65.7 \%$ reported a need for more information about infertility and options for having children [6]. This highlights the essential need for timely dissemination of reproductive health information to AYA cancer patients.

Other areas of reproductive health are also essential to AYA cancer care. Healthcare providers have reported a lack of clarity of roles within the oncologic care team for contraception education and assume that other team members addressed contraception. They also reported a lack of adequate education about contraception, which limits contraception education with AYA cancer patients. In addition to contraception counseling, AYAs with cancer note a need for better education about sexuality and intimacy [7]. A survey revealed that the majority of AYAs receiving cancer treatment wanted to receive more information about sexual health through a conversation with a healthcare provider before the start of treatment. Those who did receive counseling reported existing information was insufficient [8].

The quality and content of messages when working with this population play a vital role in the provision of emotional support, effective illness management, and health education. Allied health professionals (AHPs), specifically social workers (SWs), psychologists, nurses, and physician assistants (PAs) are in a unique position to provide this care. Educating Nurses about Reproductive Issues in Cancer Healthcare (ENRICH) was a web-based module course, developed to assist oncology nurses with communication and reproductive health education for AYAs. The ENRICH program significantly improved communication skills, knowledge, and frequency of discussion of reproductive health with AYAs [9-12]. Based on the program's success, the NCI-funded R25 Enriching Communication skills for Health professionals in Oncofertility (ECHO) program was created in order to expand user access to a variety of AHPs [13, 14]. The ECHO program has now been running for 5 years, with a goal of increasing knowledge about and skills to address reproductive health needs of AYAs with cancer via a webbased learning community for AHPs.

As the ECHO program aims to develop a national group of trained oncology AHPs who are able to address the unique reproductive health issues faced by AYAs with cancer, a significant aspect of the program is the ability for learners from a variety of disciplines to engage in the program through asking questions, sharing experiences, and reflecting with one another. This manuscript describes the overall growth of the ECHO training program and evaluates changes in learner engagement over a 5-year period.

\section{Materials and methods}

\section{Training program}

ECHO is an 8-week online training program offered annually from 2017 to 2021 (cohort 1, 2017; cohort 2, 2018; cohort 3, 2019; cohort 4, 2020; cohort 5, 2021), with continuing education credits awarded upon course completion. In efforts to improve care for AYAs with cancer and continually meet the needs of our learners, the curriculum is reviewed and updated with each cohort based on the feedback received through the program evaluation form. Learners are surveyed at the end of the course and every year thereafter to obtain feedback on the course as well as how they have applied what they learned into everyday practice. The feedback is summarized and discussed with ECHO expert faculty at an annual meeting. Based on these discussions, the curriculum is then revised during the timeframe between cohorts. Changes to the curriculum are typically modified between cohorts and not within an active cohort.

The learning objectives of the ECHO program are to: (1) refine and implement the ECHO program to facilitate knowledge about and skills to address reproductive health needs of AYA oncology patients via a web based learning community; (2) evaluate the impact of the program at the learner (e.g., knowledge, practice behavior) and workplace level (e.g., policies, referral systems); and (3) develop a network of trained AHPs to share experiences, resources, and dissemination of the program and content.

The training program curriculum includes required and optional coursework. One required element is to view 9 learning modules in an 8-week period, each typically consisting of a pre- and post-case study, pre-recorded lecture, and assigned readings. The case studies are interactive and provide real-time feedback on actions learners choose throughout the cases. In addition, learners were required to participate in at least one of 5 module-specific discussion boards by posting a minimum of 2 discussion topics or reflections. Each discussion board corresponded to a single module and centered around the following reproductive health (RH) topics: RH in pre-pubertal patients; $\mathrm{RH}$ in male AYA patients; RH in female AYA patients; AYA psychosocial issues; and AYA sexual health. Within each module-specific discussion board, learners had the opportunity to initiate a new discussion topic or to reflect on another learner's existing topic. Optional course content included the opportunity to join live synchronous discussions and/or participate in the "Ask the Experts" discussion board. 


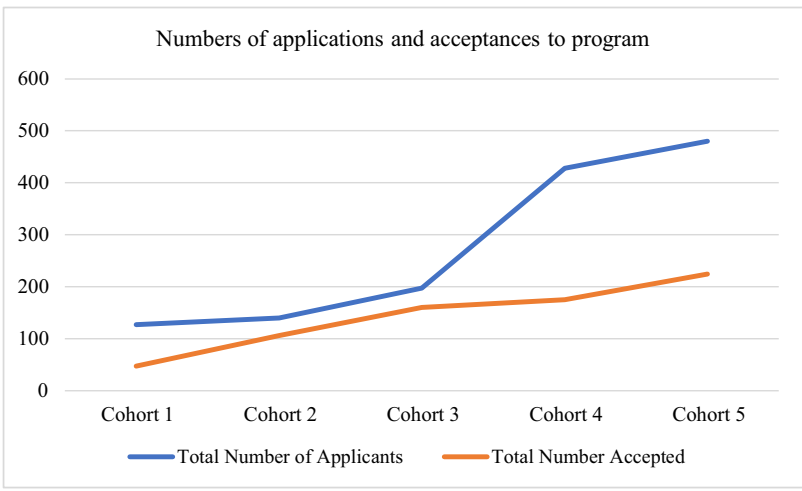

Fig. 1 Applications and acceptances to program

Synchronous discussions were live presentations conducted by 6 ECHO experts representing each of the 4 targeted learner disciplines as well as a board-certified pediatric hematologist-oncologist and a reproductive endocrinology and infertility specialist. The live synchronous virtual discussions occurred over the 8 weeks of the program. Participation was optional, and synchronous discussions were presented live as well as videotaped for those who were unable to participate at that time. Multiple synchronous discussions were never held at the same time and were almost always offered on separate dates entirely. Prior to the start of the program, the 6 presenters are allowed to select their preferred presentation data within the course dates as long as it is within the 12-3 pm EST time range in order to be reasonable for all time zones. During the first few weeks of the program, all participants are notified of the dates and times for all synchronous discussions.

The same ECHO experts who led synchronous discussions also ran Ask the Experts discussion boards, with a separate discussion board dedicated to each expert. Throughout the course, learners had the opportunity to leverage decades of experience and interact with a variety of experts in the field by posting questions for any of the experts to address.

Throughout the first 5 years of the grant, the program, ENRICH, targeted nurses. Based on this success, the reviewed grant then expanded the learners to AHPs. Prior to expanding the program, a rigorous adaptation process was completed that included an expert review panel and pilot of the curriculum with representation from targeted professions. All 4 professions in the ECHO program receive the same training. However, module 7, entitled "Communication," is a lecture tailored to the individual's profession. Individuals may choose to view all lectures for each specialty, but this is not required. For example, as a psychologist for module 7 , the learner is automatically presented with the psychologist lecture on the online platform but may choose to view the 3 other profession-tailored lectures.

\section{Recruitment}

Eligibility criteria for the ECHO program included applicants being from one of the following professions: nurses, psychologists, PAs, or SWs, and currently providing oncology care to AYA patients. Over the course of the program, learners were recruited via word of mouth, targeted electronic communications to previous ENRICH and ECHO
Fig. 2 Applications and acceptances to program by specialty

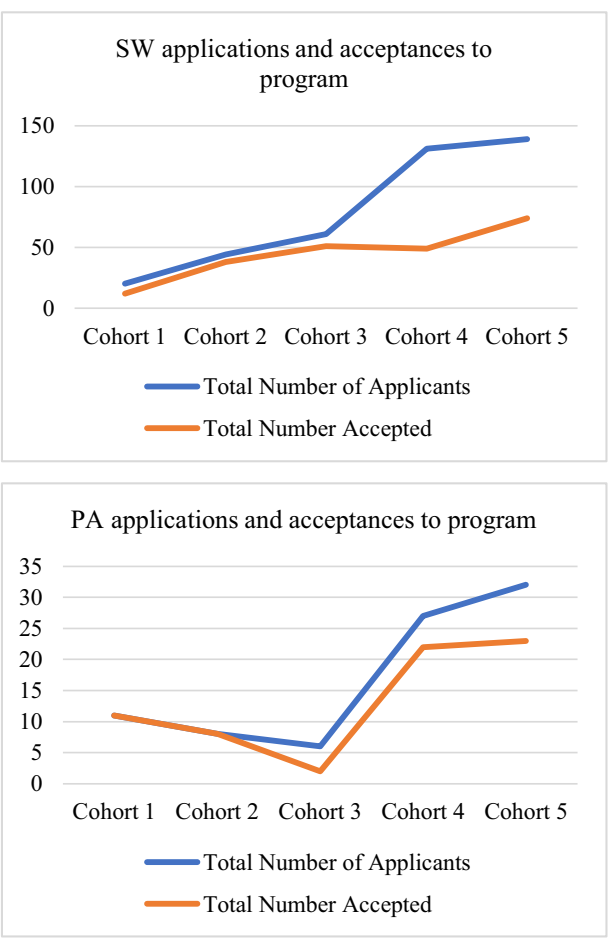




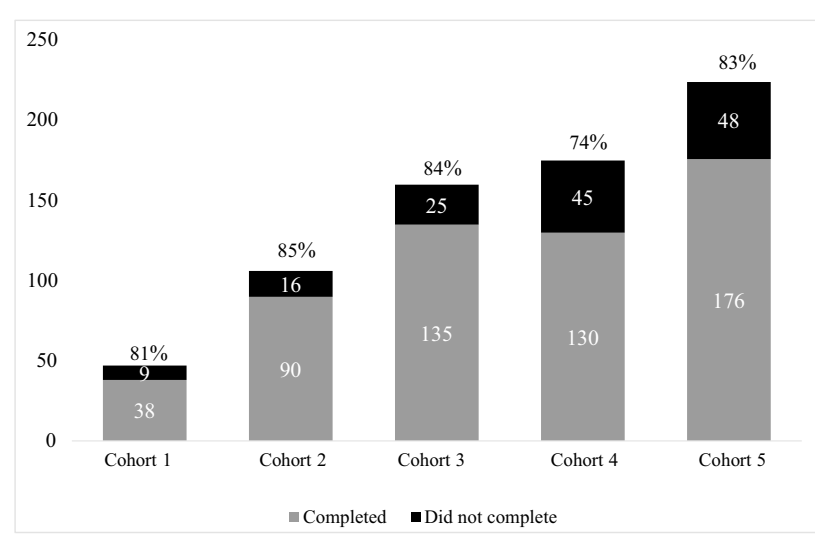

Fig. 3 Program completion

learners and 61 oncology-specific professional organizations and their special interest groups, 19 of which focus on minority AHPs. In-person recruitment strategies included promotion of $\mathrm{ECHO}$ during podium presentations at national scientific meetings given by faculty and previous learners and distribution of a training program flyer and other advertisements at local, regional, and national conferences. Interested applicants completed an online application which was reviewed and ranked by the ECHO team. Due to the increasing interest in the program and funding limitations, ECHO was unable to accept all eligible and qualified applicants. Applicants who were most likely to work with the targeted population and benefit from the program were the ones who were selected to participate.

\section{Learner engagement}

To assess learner engagement and interactions, learner reflections were evaluated. The quality of each reflection was calculated as 1 point each for mention of application of discussion topics to learner's work, a new idea, sharing a personal experience, responding to another reflection, asking a question, or reflecting on a specific quote or fact. Each of the 5 module-specific discussion boards were evaluated for number of discussion topics and reflections. Synchronous discussions were evaluated for number of questions asked by the instructor, number of patient case studies presented, number of questions asked by learners, and number of learners in attendance. Ask the Experts discussion boards were evaluated for number of unique discussion topics and reflections.

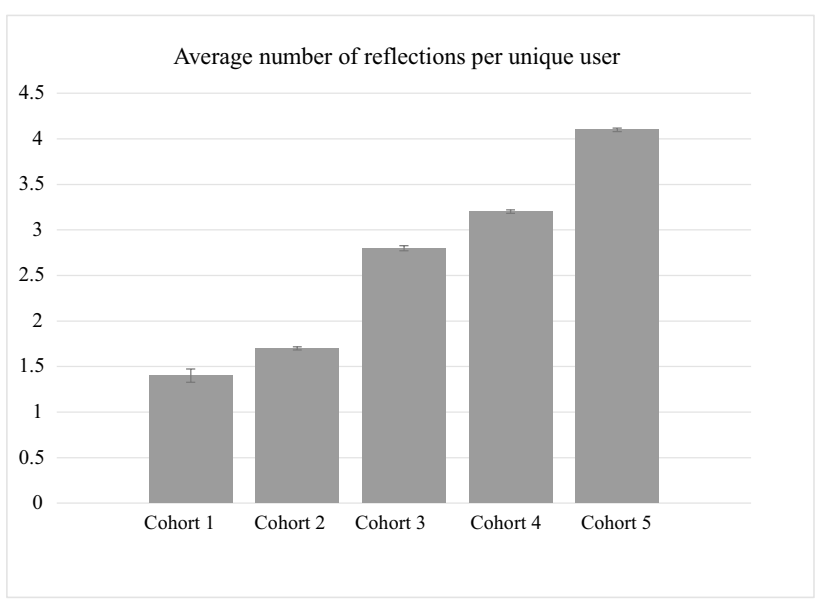

Fig. 4 Average number of reflections per unique user

\section{Data analysis}

To assess changes in quality of reflections and length of reflections, the quality score and number of words were compared between the 5 cohorts using analysis of variance (ANOVA) with a $p<0.05$ level of significance. Descriptive statistics summarized learner demographics, module topics and reflections, and synchronous discussions. Data were analyzed using IBM SPSS Statistics 27.0 [15].

\section{Results}

\section{Recruitment and retention}

Between 2017 and 2021, there were 5 cohorts of learners. The total number of applications to the program increased each year, starting with 127 applicants in cohort 1 and most recently 480 applicants in cohort 5 . Program acceptances increased each year as well, with 47 applicants accepted in cohort 1 and most recently 224 applicants accepted in cohort 5 (Fig. 1). The breakdown of applicants and accepted learners, by specialty, is provided in Fig. 2. The majority of learners completed the program in all 5 cohorts, with the highest completion rate occurring in cohort 2 , at $85 \%$ (Fig. 3).

\section{Learner engagement}

\section{Modules}

There were a total of 171 discussion topics in cohort 4 and 165 discussion topics in cohort 5 , across all modules. Within each discussion topic, the average number of reflections per unique learner increased from cohort 1 to cohort 5 , with an 


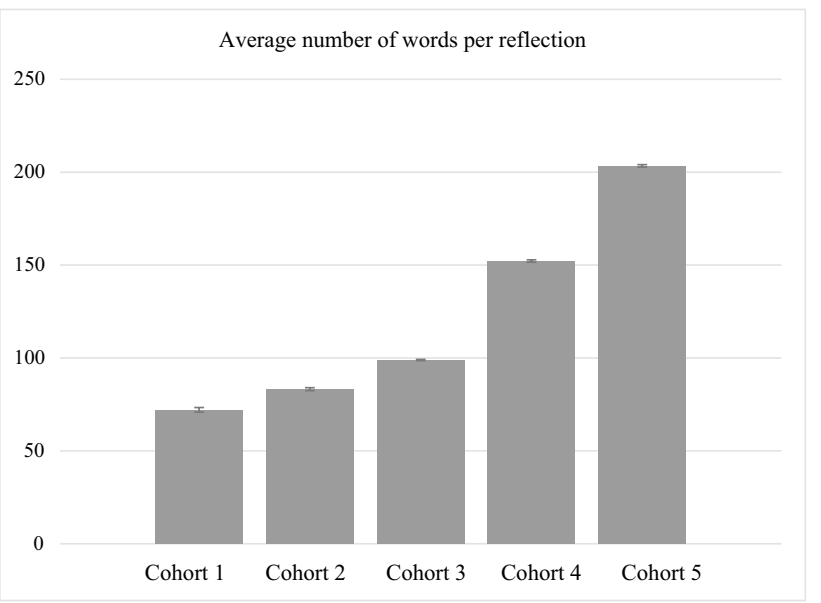

Fig. 5 Average number of words per reflection

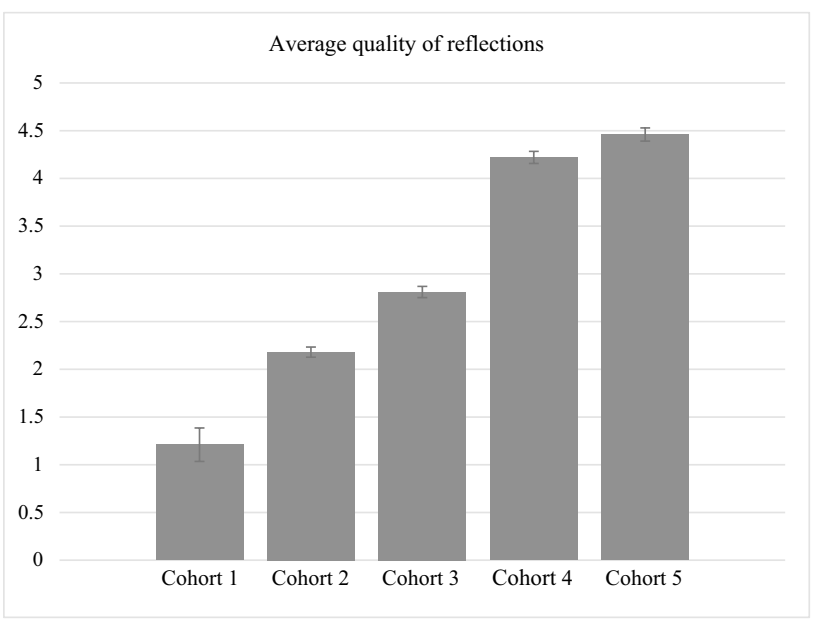

Fig. 6 Average quality of reflections average of 1.4 reflections per user in cohort 1 and 4.1 reflections per user in cohort 5 (Fig. 4).

In cohort 4 , the module on reproductive health and male AYA patients had the most discussion topics and reflections (50 topics, 119 reflections). In cohort 5, the module on reproductive health in pre-pubertal patients had the most topics and reflections (39 topics, 132 reflections). The average length of reflections and average quality of reflections significantly increased from cohort 1 to cohort 5 as well. The average length of a reflection in cohort 1 was 72.1 words, which increased to 203.4 words in cohort $5, p<0.0001$ (Fig. 5). The average quality of a reflection in cohort 1 had a score of 1.21 , which increased to a score of 4.46 in cohort $5, p<0.0001$ (Fig. 6).

\section{Synchronous discussions}

The percentage of learners in attendance at the synchronous discussions increased between cohorts 4 and 5 . An average of $4.8 \%$ of total learners were present across the 6 synchronous discussions in cohort 4 , while an average of $18.8 \%$ of total learners were present across the 6 synchronous discussions in cohort 5 . Learners asked the instructors a total of 10 questions in cohort 4 and a total of 43 questions in cohort 5 , with an average of 0.2 questions asked per learner in both cohorts. There were a total of 11 patient case studies presented by the instructors in both cohorts 4 and 5. The highest percentage of learners were present in the cohort 5 synchronous discussions led by ECHO's psychologist expert, on AYA sexual health, and ECHO's SW expert, on fertility preservation in AYA females with cancer, with $23.7 \%$ of learners in each. The synchronous discussion with the highest number of questions asked per learner was during cohort 5 on ethical challenges in fertility preservation, led by ECHO's PA expert, with an average of 0.4 questions asked per learner present (Fig. 7).

Fig. 7 Synchronous discussions

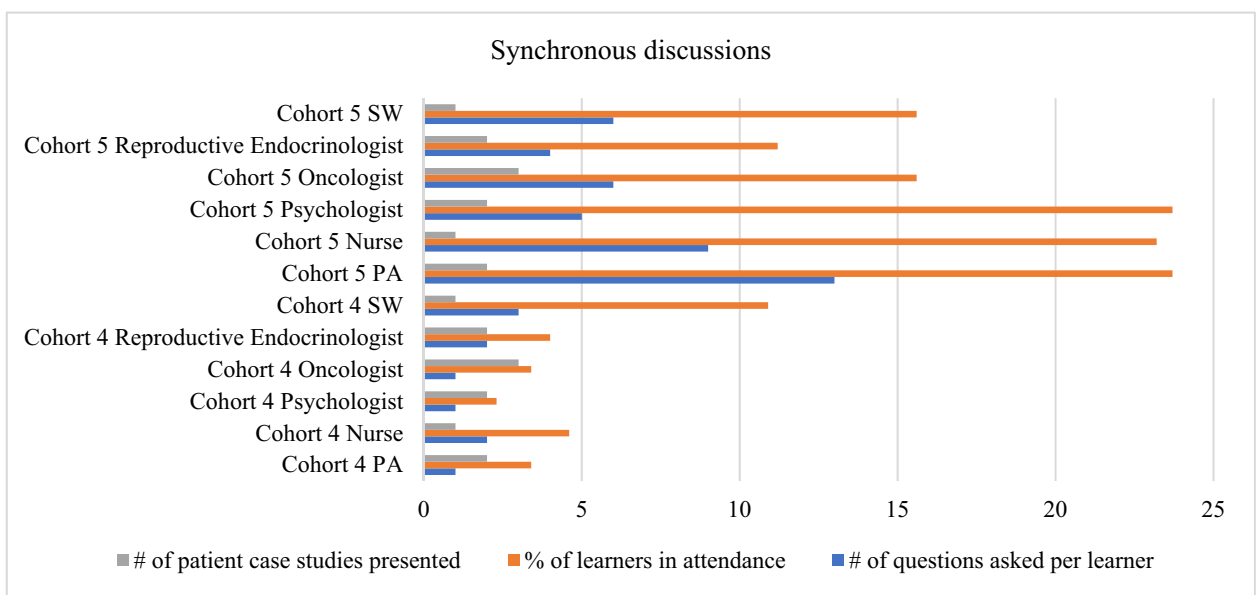




\section{Ask the Experts}

The number of Ask the Experts topics increased from 8 in cohort 4 to 16 in cohort 5. Similarly, the total number of Ask the Experts questions and reflections increased from 5 in cohort 4 to 33 in cohort 5 . In cohort 4 , the pediatric oncology nurse expert had the most topics and reflections ( 2 topics, 2 reflections), while in cohort 5, the oncology PA expert had the most topics (4), and the pediatric oncology nurse expert had the most reflections (12).

\section{Discussion}

The ECHO program has seen significant growth over a 5-year period, and it serves as an important training resource for AHPs working in oncology care with AYA patients. This contributes to the larger objective of the ECHO program grant, which was to educate professionals in effectively communicating with AYA cancer patients in all aspects of reproductive health. To our knowledge, this is the first provider-focused intervention to demonstrate success in learner engagement with Oncofertility learning modules.

Technology is reshaping the educational landscape, and online module programs offer one avenue of enhancing an education experience, overcoming geographic limitations, and providing greater learner flexibility. This is especially useful during the current uncertain times of learning during a pandemic. While some studies suggest online programs may contribute to isolation of learners, we found learners with each successive cohort to be increasingly present and engaged in modules [16]. This is particularly important given that student learning outcomes in online courses can be predicted by the level of engagement with online content [17]. The ECHO program is exceeding its goal of promoting collaborative learning among a variety of disciplines for working within the oncofertility context. This has important clinical implications for the future direction of the ECHO program and for colleagues considering developing and testing similar programs.

As the program's growth and learner engagement steadily improved year after year, it is clear that this is a unique learning environment and community that meets an important need for training in oncofertility among the oncology workforce.

A limitation of this study was that the ECHO program made changes in the way it was conducted between cohorts 1-3 and cohorts 4-5. Accessibility to full data in cohorts 1-3 was limited, and the differences between program structure in cohorts made the information challenging to compare completely. Complete data on all 5 cohorts was collected in terms of curriculum requirements (completion and results of pre/post-test, modules 1-9 pre/post case study, lectures, 2 assignments, program evaluation). This also includes data for all cohorts for the module discussion boards. All of the data that was not collected for cohorts $1-3$ is what was considered optional course content (synchronous discussions and ask the experts), and these components were developed and improved throughout the 5 years based on participant and external reviewer feedback. In cohort 4, there was also a change in management, with a new lead coordinator and new program assistant, leading to implementation of improved systematic tracking of optional course content.

Some notable changes in program structure starting with cohort 4 include the addition of the Ask and Expert discussion board, requiring learners to create at least 2 discussion topics or reflections throughout the course, and modification of the course requirement deadline schedule. Based on learner feedback, deadlines for assignments and course requirements changed so that course requirements only needed to be completed by the last day of the program, thus providing learners with more flexibility. It is possible that this change contributed to subsequent increases in learner engagement. Though the addition of the Ask an Expert discussion board is optional, it serves to encourage interaction and collaboration among AHPs, both between colleagues and subject matter experts. The method of signing up for synchronous discussions also changed between cohorts 4 and 5 . In the past, learners would be required to sign up to attend the synchronous discussion weeks in advance and would only receive the online link if signed up. In cohort 5, learners were not required to sign up to attend, and instead, all learners received an email reminder on the day of the synchronous discussion along with the link. It should also be noted that cohort 4 was ongoing during the start of the COVID-19 pandemic. As many learners are AHPs on the front lines, this may have impacted their ability to successfully participate in the program.

\section{Conclusion}

AHPs are in a unique position to provide reproductive healthcare to AYAs with cancer, and the ECHO program provides a successful method for training professionals in this area. We found that since its start in 2017, the ECHO training program has grown steadily and has had an increase in learner engagement through asking questions and sharing experiences. These educational gains then translate into the multidisciplinary care of AYAs with cancer with a focus on reproductive health. As fertility and reproductive health remain a top life goal and discussion priority for AYAs surviving cancer, increasing clinical competencies of AHPs in oncofertility is essential. 
Author contribution All authors contributed substantially to the project and manuscript.

Data availability Not applicable.

Code availability Not applicable.

\section{Declarations}

Ethics approval Not applicable.

Consent to participate Not applicable.

Consent for publication Not applicable.

Conflict of interest The authors declare no competing interests.

\section{References}

1. Kim, et al. How can health professionals enhance interpersonal communication with adolescents and young adults to improve health care outcomes? Systematic literature review. Int J Adolesc Youth. 2018;23(2):198-219.

2. Pennant et al. The role of social support in adolescent/young adults coping with cancer treatment. Children. 2019; 7(2).

3. Klosky, et al. Fertility as a priority among at-risk adolescent males newly diagnosed with cancer and their parents. Support Care Cancer. 2015;23(2):333-41.

4. Quinn, et al. Coping styles of female adolescent cancer patients with potential fertility loss. J Adolesc Young Adult Oncol. 2013;2:66-71.

5. Gorman, et al. How do you feel about fertility and parenthood? The voices of young female cancer survivors. J Cancer Surviv. 2021;6(2):200-9.

6. Zebrack B. Information and service needs for young adult cancer patients. Support Care Cancer. 2008;16:1353-60.

7. Lindsay $\mathrm{S}$, et al. Understanding barriers to contraception screening and referral in female adolescents and young adults with cancer. J Adolesc Young Adult Oncol. 2020;9(1):63-71.
8. Albers L et al. Exploring communication about intimacy and sexuality: what are the preferences of adolescents and young adults with cancer and their health care professionals? J Adolesc Young Adult Oncol. 2020; 9(2).

9. Quinn GP, Woodruff TK, Knapp CA, Bowman ML, Reinecke J, Vadaparampil ST. Expanding the oncofertility workforce: training allied health professionals to improve health outcomes for adolescents and young adults. J Adolesc Young Adult Oncol. 2016;5(3):292-6.

10. Quinn, et al. Impact of a web-based reproductive health training program: ENRICH (Educating Nurses about Reproductive Issues in Cancer Healthcare). Psychooncology. 2019;28(5):1096-101.

11. Vadaparampil, et al. ENRICH: A promising oncology nurse training program to implement ASCO clinical practice guidelines on fertility for AYA cancer patients. Patient Educ Couns. 2016;99(11):1907-10.

12. Quinn G, Vadaparampil S. ECHO: Enriching communication skills for health professionals in oncofertility training. Invited oral presentation at the 2019 International Society of Psychosomatic Obstetrics and Gynecology, The Hague, Netherlands (10/19)

13. Gwendolyn Quinn, Meghan Bowman, Ivana Sehovic, Joanne Kelvin, Clement Gwede, Cathy Meade, Joyce Reinecke, Richard Reich, Susan Vadaparampil, and the ENRICH Training Group* Impact of the ENRICH (Educating Nurses about Reproductive Issues in Cancer Healthcare) Web- Based Training Program. Podium Presentation at the 2015 World Congress on Psychooncology. Washington, D.C

14. Bowman-Curci, M., Quinn, G.P., Vadaparampil, S.T., ECHO Working Group. Impact of Enriching Communication Skills for Health Professionals in Oncofertility (ECHO): a web-based training program for health professionals. Poster presentation at the Oncofertility Consortium Conference, Chicago, IL. (2017, November).

15. IBM Corp. Released 2020. IBM SPSS Statistics for Windows, Version 27.0. Armonk, NY: IBM Corp.

16. Reeves et al. Interprofessional online learning for primary healthcare: findings from a scoping review. BMJ Open. 2017; 7(8).

17. Green, et al. The relationship between student engagement with online content and achievement in a blended learning anatomy course. Am Assoc Anat. 2018;11(5):471-7.

Publisher's note Springer Nature remains neutral with regard to jurisdictional claims in published maps and institutional affiliations. 